\title{
Ultrastructural Analysis of IpaD at the Tip of the Nascent MxiH Type III Secretion Apparatus of Shigella flexneri
}

\author{
Chelsea R. Epler ${ }^{1}$, Nicholas E. Dickenson ${ }^{1}$, Esther Bullitt ${ }^{2}$, and Wendy L. Picking ${ }^{1}$ \\ ${ }^{1}$ Department of Microbiology and Molecular Genetics, Oklahoma State University, Stillwater, \\ OK, USA \\ ${ }^{2}$ Department of Physiology and Biophysics, Boston University School of Medicine, Boston, MA, \\ USA
}

Shigella flexneri is a Gram-negative bacterium that is a significant cause of dysentery after ingestion of only 10-200 bacteria. Shigella uses its type III secretion system (T3SS) to deliver proteins into target cells, hijacking normal cellular functions. The type III secretion apparatus (T3SA) consists of a basal body and an extracellular needle. Nascent T3SA needles have at their tip the Invasion plasmid antigen $\mathrm{D}$ (IpaD). The presence of $\mathrm{IpaD}$ controls tip secretion and acts as an environmental sensor for triggering recruitment of the first translocator protein to the bacterial surface. Our hypothesis was that IpaD is situated at the needle tip alone and thereby blocks subsequent translocators as well as secretion. To test this hypothesis, we set out to determine the structure of the nascent T3SA needle tip, and verify its components.

The available IpaD crystal structure [1] could not be modeled at the tip of the T3SA such that it allowed passage of translocators and effectors. Here we use transmission electron microscopy (TEM) and EMAN2 (Electron Microscopy ANalysis Software) to produce the first threedimensional (3D) reconstruction of wild type $\mathrm{IpaD}, \mathrm{IpaD}^{\Delta 192-267}$, and an IpaD-null needle tip from $S$. flexneri. We show that wild type IpaD sits atop the T3SA needle tip as an elongated homopentamer [2]. The central coiled coil of IpaD is situated $20^{\circ}$ from the needle axis, the distal domain is elongated, and the N-terminal domain is proximal to the central coiled coil. Furthermore, we show that the distal domain, which is necessary to prevent hyper-secretion of effector proteins, is positioned to block the central channel of the T3SA needle.

Bacterial cultures: S. flexneri were grown to late-log phase in TSB complemented with $0.2 \%$ arabinose, harvested and resuspended in $2 \%$ glutaraldehyde. Needle shearing: Bacterial suspension was pushed through a $21 \mathrm{G}$ needle to allow the removal of T3SA needles by shear force. Grid Prep: Needles are laid on carbon-coated 300 mesh copper grids. Samples are washed with Tris-EDTA buffer and stained with 1\% uranyl acetate. Transmission Electron Microscopy (TEM): Grids were imaged onto film using a Philips transmission electron microscope at an electron acceleration voltage of $100 \mathrm{kv}$ and a magnification of $60,000 \mathrm{x}$. Tobacco Etch Virus (TEV) cleavage: TEV was added to an unfixed bacteria resuspended in PBS and allowed to incubate for $30 \mathrm{~min}$ at $32^{\circ} \mathrm{C}$ followed by $30 \mathrm{~min}$ at $37^{\circ} \mathrm{C}$. Culture supernatant was collected and proteins were precipitated with TCA and analyzed by western blot. 


\section{References}

[1] Johnson, S., Roversi, P., Espina, M., Olive, A., Deane, J. E., Birket, S. et al. (2007). Selfchaperoning of the type III secretion system needle tip proteins IpaD and BipD. J. Biol. Chem. 282, 4035-4044.

[2] Epler, C. R., Dickenson, N. E., Bullitt, E. \& Picking, W. L. (2012). Ultrastructural analysis of $\mathrm{IpaD}$ at the tip of the nascent MxiH type III secretion apparatus of Shigella flexneri. $J$ Mol Biol 420, 29-39.
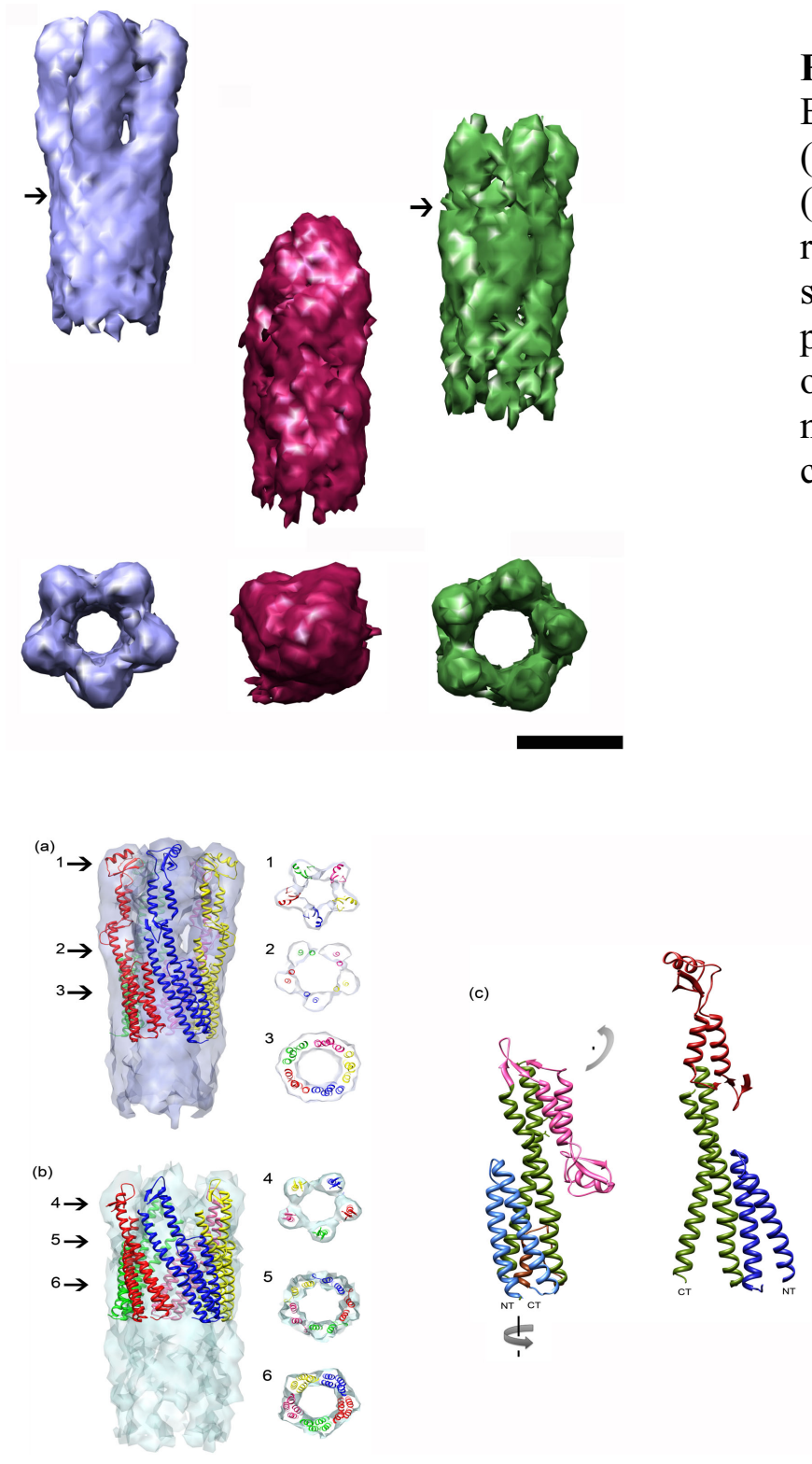

Figure 1. Type III Secretion Apparatus Tips. Electron density maps of wild-type IpaD (blue), ipaD-null (magenta), and $\mathrm{IpaD}^{\Delta 192-267}$ (green) are shown at $20 \AA, 24 \AA$ and $22 \AA$ resolution, respectively. The lower panels show the reconstructions tilted $90^{\circ}$ in the positive $z$ plane to show the constricted opening of wild-type IpaD and ipaD-null needle ends. The black arrows indicate the collar region and the scale bar is $50 \AA$.
Figure 2. Modeling the IpaD Crystal Structure into Electron Density Maps. To find a satisfactory fit the crystal structure of IpaD had to be elongated by shifting the distal domain upward (c, pink to red). The central coiled coil fits at a $20^{\circ}$ tilt from the needle axis while the N-terminal domain pivots proximal to the central coiled coil ( $\mathrm{c}$, light blue to blue). The numbers and arrows are highlighting $15-\AA \AA$ slices to show the fit. Wild-type IpaD (a) was modeled with full length IpaD while $\mathrm{IpaD}^{\Delta 192-267}$ (b) was modeled with IpaD minus residues 192-267. 